\title{
Improved stability estimates and a characterization of the native space for matrix-valued RBFs
}

\section{Edward J. Fuselier}

Published online: 7 August 2008

(C) Springer Science + Business Media B.V. 2008

\section{Erratum to: Adv Comput Math DOI 10.1007/s10444-007-9046-3}

Unfortunately, there was an error in the proof of Theorem 6 of [2]. In this paper a corrected proof is presented.

We will use $\|\alpha\|_{2}$ to denote the standard Euclidean norm of $\alpha \in \mathbb{R}^{m}$, and write $\alpha^{T}$ for the transpose of $\alpha$. We will use the following convention for the Fourier transform and inverse Fourier transform of a function:

$$
\widehat{f}(\xi):=\int_{\mathbb{R}^{n}} f(x) e^{-i x^{T} \xi} d \xi, \quad \check{f}(x):=\frac{1}{(2 \pi)^{n}} \int_{\mathbb{R}^{n}} f(\xi) e^{i \xi^{T} x} d \xi .
$$

Let $X=\left\{x_{1}, \ldots, x_{N}\right\} \subset \mathbb{R}^{n}$ be a set of distinct points. We let $q_{X}$ denote the separation radius of $X$, which is defined by

$$
q_{X}:=\frac{1}{2} \min _{i \neq j}\left\|x_{i}-x_{j}\right\| .
$$

We will say an $m \times m$ matrix-valued function $\Phi$ is positive definite on $\mathbb{R}^{n}$ if given any finite, distinct set of points $X$, we have

$$
\sum_{j, k=1}^{N} \alpha_{j}^{T} \Phi\left(x_{j}-x_{k}\right) \alpha_{k} \geq 0
$$

for all $\alpha_{1}, \ldots, \alpha_{N}$ in $\mathbb{R}^{m}$. This is equivalent to saying that the $m N \times m N$ matrix whose $(j, k)^{t h} m \times m$ block is given by $\Phi\left(x_{j}-x_{k}\right)$ is positive definite. This matrix

The online version of the original article can be found under doi:10.1007/s10444-007-9046-3.

E. J. Fuselier $(\varangle)$

Department of Mathematical Sciences, United States Military Academy,

West Point, NY 10996, USA

e-mail: edward.fuselier@usma.edu 
will be denoted by $A_{X, \Phi}$. If a positive definite function $\phi: \mathbb{R}^{n} \rightarrow \mathbb{R}$ is radial, i.e. $\phi(x)=\phi\left(\|x\|_{2}\right)$, then we call $\phi$ a radial basis function $(\mathrm{RBF})$.

Given an RBF $\phi$ defined on $\mathbb{R}^{n}$, one can construct the matrix-valued function $\Phi_{d i v}=\left(-\Delta+\nabla \nabla^{T}\right) \phi$. This function is positive definite, and its columns are divergence-free [1]. The stability of the matrix $A_{X, \Phi_{d i v}}$ has been studied, and recently the following result was presented [2, Theorem 6].

Theorem 6 Let $\phi$ be an even positive definite function, which possesses a positive Fourier transform $\widehat{\phi} \in C\left(\mathbb{R}^{n} / 0\right)$. With the function

$$
M(\sigma):=\inf _{\|\xi\|_{2} \leq \sigma} \widehat{\phi}(\xi)
$$

a lower bound on the smallest eigenvalue of the interpolation matrix is given by

$$
\lambda_{\min }\left(A_{X, \Phi_{d i v}}\right) \geq\left(\frac{\sigma^{2}}{16 \pi}\right)^{(n+2) / 2} \frac{M(\sigma) \pi}{(4 \pi)^{n} \Gamma((n+2) / 2)}
$$

for any $\sigma>0$ satisfying

$$
\sigma \geq \widetilde{C} / q_{X}
$$

where $\widetilde{C}$ is a constant independent of $\phi$ and $X$.

It has come to the attention of the author that there was an error in the proof. Below is a corrected version.

Proof To condense our notation, we define the constant

$$
C_{\sigma}:=\frac{M(\sigma) \Gamma((n+2) / 2)}{\sigma^{n} \pi^{n / 2}}
$$

Let $\chi_{\frac{\sigma}{2}, n}(\xi)$ be the characteristic function for the ball $B(0, \sigma / 2) \subset \mathbb{R}^{n}$, and define $\psi$ by $\psi:=C_{\sigma} \check{\chi}_{\frac{\sigma}{2}, n}^{2}$. Note that $\psi$ is positive definite and the support of $\widehat{\psi}$ is in $B(0, \sigma)$. It follows then that $\widehat{\phi}(\xi) \geq \widehat{\psi}(\xi)$ for $\|\xi\|_{2}>\sigma$. If $\|\xi\|_{2} \leq \sigma$, we have

$$
\widehat{\psi}(\xi) \leq C_{\sigma} \operatorname{vol}(B(0, \sigma)) \leq M(\sigma) \leq \widehat{\phi}(\xi)
$$

This shows us that

$$
\begin{aligned}
\sum_{j, k=1}^{N} \alpha_{j}^{T} \Phi_{d i v}\left(x_{j}-x_{k}\right) \alpha_{k} & =(2 \pi)^{-n} \int_{\mathbb{R}^{n}}\left\|\left(I-e_{\xi} e_{\xi}^{T}\right)\left(\sum_{j=1}^{N} \alpha_{j} e^{i \xi^{T} x_{j}}\right)\right\|_{2}^{2}\|\xi\|_{2}^{2} \widehat{\phi}(\xi) d \xi \\
& \geq(2 \pi)^{-n} \int_{\mathbb{R}^{n}}\left\|\left(I-e_{\xi} e_{\xi}^{T}\right)\left(\sum_{j=1}^{N} \alpha_{j} e^{i \xi^{T} x_{j}}\right)\right\|_{2}^{2}\|\xi\|_{2}^{2} \widehat{\psi}(\xi) d \xi \\
& =\sum_{j, k=1}^{N} \alpha_{j}^{T} \Psi_{d i v}\left(x_{j}-x_{k}\right) \alpha_{k},
\end{aligned}
$$

where $\alpha_{j} \in \mathbb{R}^{n}$ and $\Psi_{\text {div }}$ is defined by $\Psi_{\text {div }}=\left(-\Delta+\nabla \nabla^{t}\right) \psi$.

From the calculations done preceding equation (11) in [2, Section 4], we see that

$$
\Psi_{d i v}(0)=C_{\sigma}(n-1) A(0) I,
$$


where $A(0)$ is given by

$$
A(0)=\left(\frac{\sigma^{2}}{16 \pi}\right)^{n+1} \frac{8 \pi}{(n+2) \Gamma^{2}((n+2) / 2)} .
$$

Now we use [2, Lemma 3] to get:

$$
\begin{aligned}
\sum_{j, k=1}^{N} \alpha_{j}^{T} \Psi_{d i v}\left(x_{j}-x_{k}\right) \alpha_{k} & \geq\|\alpha\|_{2}^{2} C_{\sigma}(n-1) A(0)-\sum_{j \neq k}\left|\alpha_{j}^{T} \Psi_{d i v}\left(x_{j}-x_{k}\right) \alpha_{k}\right| \\
& \geq\|\alpha\|_{2}^{2} C_{\sigma}(n-1) A(0)-n\|\alpha\|_{2}^{2} \max _{k} \sum_{j \neq k} C_{\sigma} \Lambda\left(x_{j}-x_{k}\right) \\
& =\|\alpha\|_{2}^{2}\left(C_{\sigma}(n-1) A(0)-C_{\sigma} \frac{n-1}{2} A(0)\right) \\
& =\|\alpha\|_{2}^{2} \frac{1}{2} C_{\sigma}(n-1) A(0),
\end{aligned}
$$

where $\Lambda$ is defined in [2, Equation (13)]. The result now follows by rewriting $C_{\sigma}$ and $A(0)$ to get

$$
\begin{aligned}
\lambda_{\min }\left(A_{X, \Phi_{d i v}}\right) & \geq \frac{1}{2} C_{\sigma}(n-1) A(0) \\
& =\frac{1}{2}\left(\frac{M(\sigma) \Gamma((n+2) / 2)}{\sigma^{n} \pi^{n / 2}}\right)(n-1)\left(\left(\frac{\sigma^{2}}{16 \pi}\right)^{n+1} \frac{8 \pi}{(n+2) \Gamma^{2}((n+2) / 2)}\right) \\
& =\left(\frac{(n-1)}{2(n+2)}\right) \frac{M(\sigma)}{\sigma^{n} \pi^{n / 2}}\left(\frac{\sigma^{2}}{16 \pi}\right)^{n+1} \frac{8 \pi}{\Gamma((n+2) / 2)} \\
& \geq \frac{M(\sigma)}{\sigma^{n} \pi^{n / 2}}\left(\frac{\sigma^{2}}{16 \pi}\right)^{n+1} \frac{\pi}{\Gamma((n+2) / 2)} \\
& =\left(\frac{\sigma^{2}}{16 \pi}\right)^{(n+2) / 2} \frac{M(\sigma) \pi}{(4 \pi)^{n} \Gamma((n+2) / 2)} .
\end{aligned}
$$

Here we have used the fact that $(n-1) /(n+2) \geq 1 / 4$ for $n \geq 2$.

Acknowledgements The author would like to thank Stefan Schrödl for kindly pointing out the mistake.

\section{References}

1. Narcowich, F.J., Ward, J.D.: Generalized Hermite interpolation via matrix-valued conditionally positive definite functions. Math. Comp. 63, 661-687 (1994)

2. Fuselier, E.J.: Improved stability estimates and a characterization of the native space for matrixvalued RBFs. Adv. Comp. Math. (2007). doi:10.1007/s10444-007-9046-3 